\title{
Vertical transient loading of a suction caisson in dense sand
}

\author{
B. Cerfontaine \\ ArGEnCo Department \\ University of Liege, Liege, Belgium \\ F. Collin \& R. Charlier \\ ArGEnCo Department \\ University of Liege, Liege, Belgium
}

\begin{abstract}
Suction caissons are hollow cylinders open towards the bottom currently used as anchors for deep water offshore facilities. They recently turned out to be advantageously exploited as foundation for offshore wind turbines in shallow water (Senders 2009). The Prevost model for cohesionless soils (Prevost 1985) is adapted for the modelling of their cyclic behaviour. It is able to reproduce plastic deformation in both loading and unloading, contractancy of the soil and pore pressure build up as well. In this paper, a fully-coupled transient axisymmetric analysis of a suction caisson is carried out. The monotonic partially drained behaviour of the caisson is firstly highlighted. Afterwards, pseudo-random and sinusoidal-equivalent storm signals are compared. Permanent displacements accumulated at the end of the storm show a good agreement between them but are slightly divergent, which indicates that the position of the extreme event might be a relevant issue.
\end{abstract}

\section{INTRODUCTION}

Nowadays offshore power plants are gathering momentum. The design of their foundations is a crucial issue since their cost is non negligible (Byrne and Houlsby 2002). Classically used as anchors for deep water structures, the suction caissons are promising for shallow foundation (Houlsby et al. 2005, Stuyts et al. 2011) either in sand or clays. These hollow cylinders open towards the bottom are installed into the soil by pumping water inside, which creates a differential pressure that plugs them. They are quickly and cheaply installed (Senders 2009), easily removed and provide a limited resistance in tension by suction (Byrne and Houlsby 2002).

The Prevost's model for cohesionless soils, (Prevost 1985), is adapted to the modelling of their cyclic behaviour and is used in earthquake community (Yang and Elgamal 2008). It's able to capture plasticity effects in both loading and unloading, pore water pressure (PWP) generation and cyclic mobility.

In this paper a fully coupled transient axisymmetric analysis is carried out on a suction caisson. Firstly, a monotonic push test highlights the partially drained behaviour of the soil. The effect of signal type on PWP accumulation and displacement is secondly performed. Pseudo-random and equivalent signals lead to similar final results.

\section{EQUATIONS OF THE PREVOST MODEL}

\subsection{Definitions}

The sign convention of soil mechanics is adopted: compressive stresses and strains are positive. The Macauley brackets \langle\rangle are defined according to

$\langle\beta\rangle=\frac{1}{2} \cdot(\beta+|\beta|)$

in which the symbol ":" indicates a dot product between two tensors (in bold characters). For example, if $\sigma$ is the effective (Cauchy) stress tensor, the product $\boldsymbol{\sigma}: \boldsymbol{\sigma}=\sigma_{i j} \cdot \sigma_{i j}$ in index notation. The identity tensor is written $\delta$, then the mean effective stress is defined as $\mathrm{p}=1 / 3 \cdot \boldsymbol{\sigma}: \boldsymbol{\delta}$. The deviatoric stress tensor and the invariant of deviatoric stresses are defined through

$\mathbf{s}=\boldsymbol{\sigma}-\mathrm{p} \cdot \boldsymbol{\delta} \quad$ and $\quad \mathrm{q}=\sqrt{\frac{3}{2} \cdot \mathbf{s}: \mathbf{s}}$

\subsection{Constitutive equations}

The Prevost model lies within the framework of elasto-plasticity. Constitutive equations are written in incremental form. The equation (3) links the effective stress rate $\dot{\boldsymbol{\sigma}}$ to the elastic deformation rate $\dot{\boldsymbol{\epsilon}}-\dot{\boldsymbol{\epsilon}}^{p}$

$\dot{\boldsymbol{\sigma}}=\mathbf{E}:\left(\dot{\boldsymbol{\epsilon}}-\dot{\boldsymbol{\epsilon}}^{p}\right)$ 
where $\mathbf{E}$ is the fourth-order tensor of elastic coefficients, $\dot{\boldsymbol{\epsilon}}$ is the total deformation rate and $\dot{\boldsymbol{\epsilon}}^{p}$ is the plastic deformation rate defined through

$\dot{\boldsymbol{\epsilon}}^{p}=\mathbf{P} \cdot\langle\Delta \gamma\rangle$

in which $\mathbf{P}$ is a symmetric second-order tensor defining a non-associated plastic potential. The plastic loading function, $\Delta \gamma$, is a scalar that depicts the variation of plastic deformation and is defined in (5)

$\Delta \gamma=\frac{1}{\mathrm{H}^{\prime}} \cdot \mathbf{Q}: \dot{\boldsymbol{\sigma}}$

where $\mathbf{Q}$ is a second-order tensor defining the unit outer normal to the yield surface and $\mathrm{H}^{\prime}$ the plastic modulus associated to this surface.

\subsection{Yield functions}

The model is made of conical nested yield surfaces in principal stress space (Prevost 1985). Their apex is fixed at the origin of axes but could be translated on the hydrostatic axis to take cohesion into account if necessary. The i-th surface is the locus of the stress states that verify

$\mathrm{f}^{i} \equiv\left(\mathbf{s}-\mathbf{p} \cdot \boldsymbol{\alpha}^{i}\right):\left(\mathbf{s}-\mathbf{p} \cdot \boldsymbol{\alpha}^{i}\right)-\frac{2}{3} \cdot\left(\mathrm{p} \cdot \mathbf{M}^{i}\right)^{2}=0$

where $\boldsymbol{\alpha}^{i}$ is a kinematic deviatoric tensor defining the coordinates of the yield surface centre in deviatoric space (=backstress) and $\mathrm{M}^{i}$ is a material parameter denoting the aperture of the cone. A normal to the yield surface is computed through

$$
\begin{aligned}
\frac{\partial f}{\partial \boldsymbol{\sigma}} & =2 \cdot\left(\mathbf{s}-\mathrm{p} \cdot \boldsymbol{\alpha}^{i}\right) \\
& +\frac{2}{3} \cdot\left[\mathrm{p} \cdot\left(\boldsymbol{\alpha}^{i}: \boldsymbol{\alpha}^{i}-\frac{2}{3} \cdot\left(M^{i}\right)^{2}\right)-\mathbf{s}: \boldsymbol{\alpha}^{i}\right] \cdot \boldsymbol{\delta}
\end{aligned}
$$

A unit-norm normal tensor is then computed and can be decomposed into deviatoric and volumetric parts as

$$
\mathbf{Q}=\frac{\frac{\partial f}{\partial \boldsymbol{\sigma}}}{\left\|\frac{\partial f}{\partial \boldsymbol{\sigma}}\right\|}=\mathbf{Q}^{\prime}+\mathbf{Q}^{\prime} \cdot \boldsymbol{\delta}
$$

\subsection{Plastic flow rule}

The plastic potential $\mathbf{P}=\mathbf{P}^{\prime}+\mathrm{P}^{\prime \prime} \cdot \boldsymbol{\delta}$ is decomposed into its deviatoric part which is associative

$\mathbf{P}^{\prime}=\mathbf{Q}^{\prime}$

and its volumetric part which is non-associative

$\mathrm{P}^{\prime \prime}=\frac{1}{3} \cdot \frac{\bar{\eta}^{2}-\eta^{2}}{\bar{\eta}^{2}+\eta^{2}}$ where $\eta=\frac{\sqrt{3 / 2 \cdot \mathbf{s}: \mathbf{s}}}{\mathrm{p}}=\frac{\mathrm{q}}{\mathrm{p}}$

The material parameter $\bar{\eta}$ takes into account the phase transformation line (PTL) defined by Ishihara (Ishihara et al. 1975). This parameter rules the volumetric behaviour and separates the p-q plane into two zones. Stress ratios $(\eta)$ lower than $\bar{\eta}$ indicate a plastic contractive behaviour whilst the other zone depicts a dilative plastic behaviour.

\subsection{Hardening rule}

The hardening rule of the surfaces is purely kinematic. During loading, the active surface moves up to come into contact with the next one. The relationship between plastic function and kinematic hardening is determined through the consistency condition (Prevost 1985) and leads to

$\mathrm{p} \cdot \dot{\boldsymbol{\alpha}}^{i}=\frac{\mathrm{H}^{\prime}}{\mathbf{Q}^{\prime}: \boldsymbol{\mu}} \cdot\langle\Delta \gamma\rangle \cdot \boldsymbol{\mu}$

where $\boldsymbol{\mu}$ is a tensor defining the direction of translation of the active surface in the deviatoric space. At this step, any direction of translation could be used depending on the strategy used to integrate the constitutive law (explicit or implicit). The only requirement is that any surface has to be at most tangential to the next one, at the end of a given step. Overlapping of the surfaces is then avoided. In this paper, an implicit integration is adopted.

\section{CASE STUDY DEFINITION}

\subsection{Geometry}

The caisson modelled in the finite element code LAGAMINE is a $8 \mathrm{~m}$ diameter (D) caisson, part of a tripod foundation in shallow water (see in Figure 1a). In this case, the overturning moment created by action of waves and wind is mainly carried out by push-pull action on opposite caissons (Kelly et al. 2006). Therefore, to a first approximation, the horizontal load is neglected and the foundation can be idealized as an axisymmetric case.

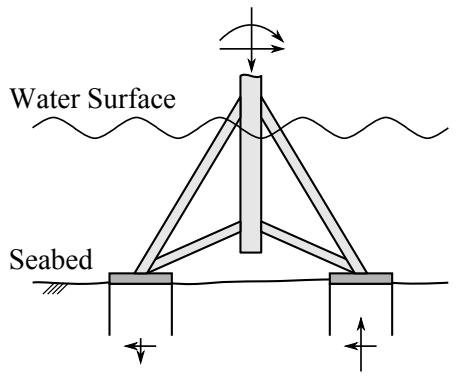

(a) Tripod foundation for wind turbine.

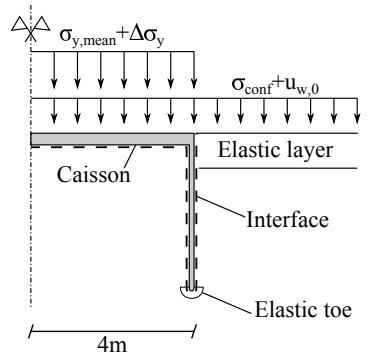

(b) Geometry of the caisson : $\mathrm{D}=8 \mathrm{~m}$
Figure 1

The total domain is a rectangle of $24 \mathrm{mx} 26 \mathrm{~m}$. A sketch of the geometry of the caisson is given in Figure 1b. (Kelly et al. 2006) report wall thickness of the caisson between $0.3 \%$ and $0.5 \%$ of D. However, for numerical purpose, the thickness adopted is $1.25 \%$ of $\mathrm{D}$, i.e. $0.1 \mathrm{~m}$. The top of the caisson is assumed very stiff in order to distribute the load more uniformly.

The first $0.8 \mathrm{~m}$ of the seabed is assumed linear elastic to avoid local failure and superficial liquefaction. Identical justification holds for the definition of an 
elastic soft toe under the tip of the caisson. The Prevost model is applied elsewhere. Special interface elements are applied on the caisson sides. They allow for a relative sliding between the soil and the caisson.

Loads applied are described in Figure $1 \mathrm{~b}: \mathrm{a}$ confinement at the top $\sigma_{\text {conf } f}$, a static water pressure $u_{w, 0}$ (the seabed is at a depth of $10 \mathrm{~m}$ ), the dead weight of the superstructure $\sigma_{y, \text { mean }}$ and a cyclic amplitude $\Delta \sigma_{y}$ due to wind and waves.

\subsection{Material}

Parameters of a synthetic dense sand are given in table 1 . The model is composed of 10 nested surfaces described by their half-opening $\mathrm{M}$, their plastic modulus $H^{\prime}$ and their backstress $\alpha$. In order to facilitate the analysis of results, the soil is deemed to have the same behaviour in compression and extension as well $(\alpha=0)$ and to be initially isotropic $\left(K_{0}=1\right)$.

A small cohesion of $5 \mathrm{kPa}$ is added for numerical purpose. Elastic parameters of the superficial layer of soil are identical. The Young moduli of the caisson skirts and elastic toe are $2 \cdot 10^{5} \mathrm{MPa}$ and $10 \mathrm{MPa}$ respectively.

Table 1: Material parameters : initial position of the surfaces $\left(\alpha=\alpha_{11}-\alpha_{33}\right)$, aperture of the surfaces $(\mathbf{M})$, plastic moduli associated $\left(\mathrm{H}^{\prime}\right)$, Young modulus (E), Poisson's ratio $(\nu)$, slope of the phase transformation line $(\bar{\eta})$, effective soil weight $\left(\gamma^{\prime}\right)$, permeability $(\mathrm{k})$, porosity (n).

\begin{tabular}{cccccc}
\hline Surf. Nb. & 1 & 2 & 3 & 4 & 5 \\
$\alpha[-]$ & 0 & 0 & 0 & 0 & 0 \\
$\mathrm{M}[-]$ & 0.015 & 0.025 & 0.045 & 0.080 & 0.150 \\
$\mathrm{H}^{\prime}[\mathrm{MPa}]$ & 450 & 350 & 250 & 150 & 100 \\
\hline Surf. Nb. & 6 & 7 & 8 & 9 & 10 \\
$\alpha[-]$ & 0 & 0 & 0 & 0 & 0 \\
$\mathrm{M}[-]$ & 0.300 & 0.425 & 0640 & 0.775 & 0.92 \\
$\mathrm{H}^{\prime}[\mathrm{MPa}]$ & 30 & 10 & 2 & 1 & 0 \\
\hline $\bar{\eta}[-]$ & \multicolumn{5}{c}{0.4} \\
\hline \multicolumn{7}{c}{$\mathrm{E}[\mathrm{MPa}]$} & 100 & $\nu[-]$ & 0.25 \\
\hline$\gamma^{\prime}\left[\mathrm{kN} / \mathrm{m}^{3}\right]$ & 10.56 & $\mathrm{k}\left[\mathrm{m}^{2}\right]$ & $10^{-12}$ & $\mathrm{n}[-]$ & 0.36 \\
\hline \multicolumn{7}{c}{}
\end{tabular}

Mechanical behaviour of the interface is ruled by the Mohr-Coulomb criterion. That is to say that a maximal shear resistance along the interface reads

$\tau_{\text {max }}=\sigma_{n}^{\prime} \cdot \tan \varphi=\sigma_{n}^{\prime} \cdot 0.3$

where $\sigma_{n}^{\prime}$ is the effective stress normal to the sliding plane and $\varphi$ is the steel-soil friction angle. If the shear stress is lower than $\tau_{\max }$ soil and caisson are considered "stuck" and move together. On the other hand, if the maximal stress is reached, both sides of the interface encounter a relative displacement. Numerical reality is a bit different due to the special treatment of these interfaces but out of the scope of this study. Interested reader should refer to (Habraken et al. 1998).

\subsection{From pseudo-random to equivalent loading}

The constant part of the vertical loading $\sigma_{y, \text { mean }}$ stands for the dead weight of the wind turbine (= $20 \mathrm{kPa}$ ). On the other hand, loading originated from waves and wind is in essence random and time dependent. Such a loading is depicted in Figure 2. It consists of a pseudo-random signal, adapted from a real case in order to well-capture the frequency content and including an extreme event $\left(\Delta \sigma_{y}=40.5 \mathrm{kPa}\right)$.
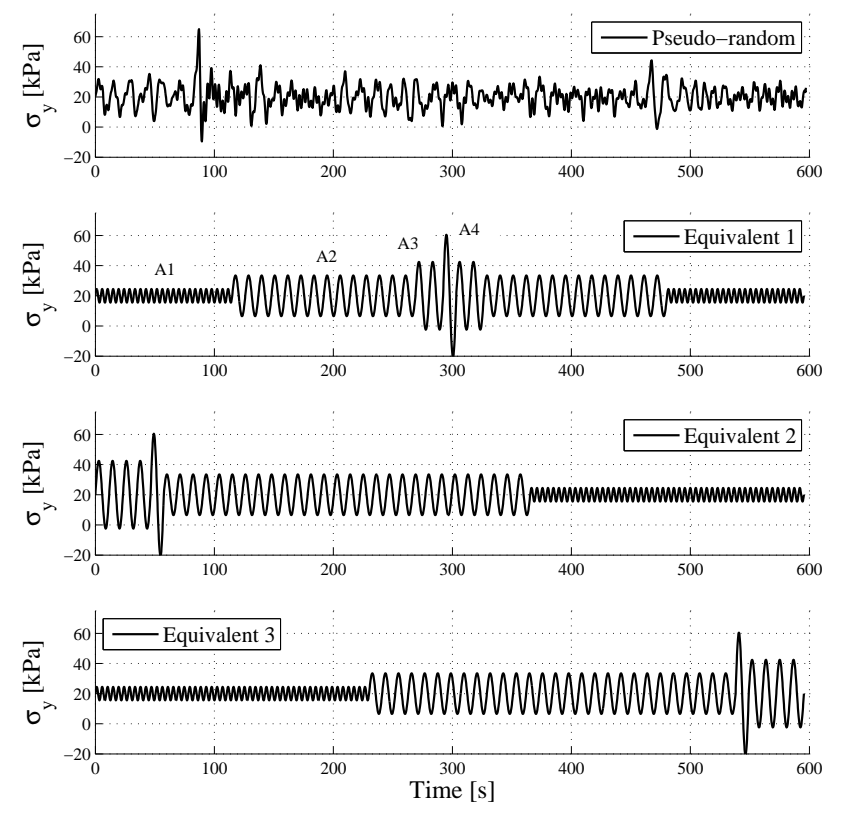

Figure 2: Pseudo-random and equivalent vertical load signals : $\sigma_{y, \text { mean }}=20 \mathrm{kPa}, V \Delta \sigma_{y}=40.5$.

Any actual signal can be transformed into an equivalent one which has an identical frequency content. A half-cycle analysis was carried out in order to transform the pseudo-random signal into sinusoidalequivalent ones ((Byrne and Houlsby 2002), see in Figure 2). The purpose of the method is to decompose the actual signal into half cycles, which are signal parts bounded by two crossings of the mean value $\sigma_{y, \text { mean }}$ (see in Figure 3). All these half cycles can be merged in batches of equivalent cycles of similar period and amplitudes and a new signal is recomposed. This definition actually filters the high frequency content of the signal. However high frequency cycles generate and dissipate nearly instantaneously few variations of pore water pressure, which has a neutral effect on the results.

In this study, results are limited to four batches of cycles associated to average amplitudes and periods (see in Table 2). Classicaly, these batches are rearranged in increasing order of amplitude up to an extreme event and decreasing amplitude afterwards, (Rahman et al. 1977). In this paper, two other rearrangements of the batches are also addressed (see in Figure 2 ). A final consolidation phase of 250 s allows for dissipating pore water pressures after the storm. 


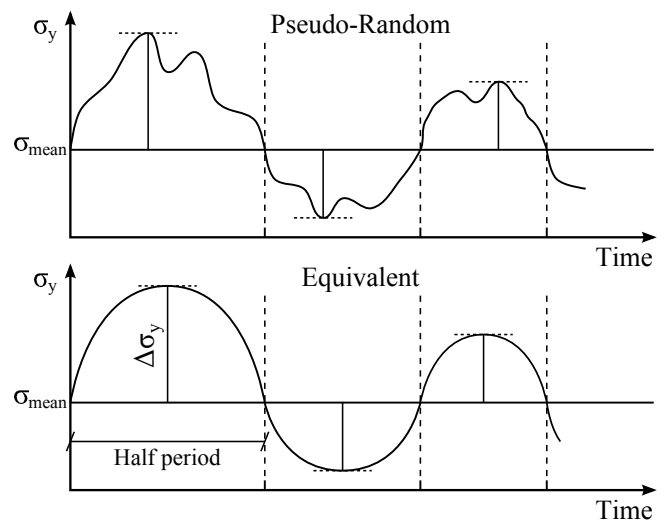

Figure 3: Half-cycle analysis.

Table 2: Number of equivalent cycles, associated amplitude and periods

\begin{tabular}{ccccc}
\hline & $\mathrm{A} 1$ & $\mathrm{~A} 2$ & $\mathrm{~A} 3$ & $\mathrm{~A} 4$ \\
Number of cycles [-] & 50 & 28 & 4 & 1 \\
$\Delta \sigma_{y}[\mathrm{kPa}]$ & 4.5 & 13.5 & 22.5 & 40.5 \\
Period $[\mathrm{s}]$ & 4.6 & 11 & 11.6 & 11.1 \\
\hline
\end{tabular}

\section{RESULTS}

\subsection{Partially drained behaviour}

Behaviour of suction caissons is inherently partially drained. That is to say that a continuously evolving equilibrium sets up between total load, PWP and effective stress. A monotonic push test is carried out in order to illustrate this phenomenon. A variation of total stress $\Delta \sigma_{y}=40.5 \mathrm{kPa}$ is progressively applied at the top of the caisson at a constant loading rate. The influence of plasticity effects is highlighted by a comparison between the aforementioned geometry and a purely elastic soil configuration.

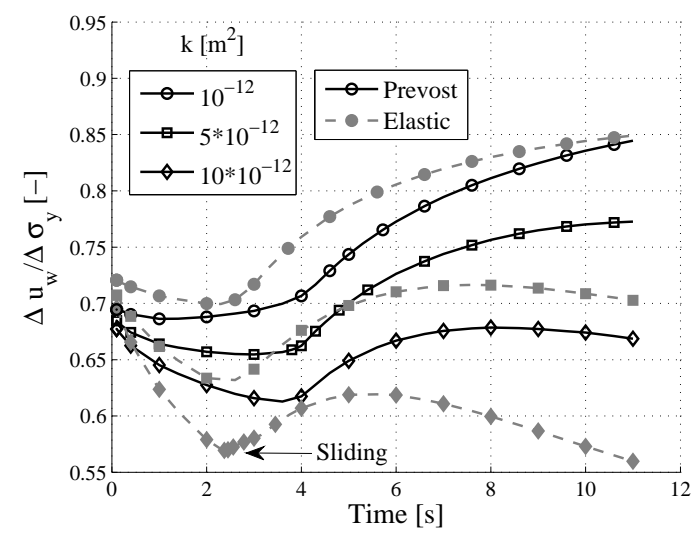

Figure 4: Monotonic push test on the caisson (empty markers $=$ Prevost model ; filled markers $=$ elastic model). $\Delta \sigma_{y}$ is the variation of loading and $\Delta u_{w}$ the pore water pressure at the top of the caisson.

The ratio of PWP generated at the top center of the caisson $\Delta u_{w}$ and the total load variation $\Delta \sigma_{y}$ at time $\mathrm{t}$ are depicted in Figure 4 for several configurations. Three permeabilities $\left(k=\left[10^{-12}, 5 \cdot 10^{-12}, 10 \cdot 10^{-12}\right] \mathrm{m}^{2}\right)$ and two constitutive laws (elastic or Prevost model) are considered.
Initially the behaviour is nearly undrained since PWP generated does not have time to dissipate. However the ratio $\Delta u_{w} / \Delta \sigma_{y}$ is not close to one but to 0.7. This paradoxical effect finds its origin in the load that is withstood by the skirts of the caisson, either by friction or end bearing. Therefore, the total load applied is redistributed between soil inside the caisson and the skirts. Obviously, the lower the permeability, the higher the ratio, since PWP dissipates more slowly, which can be observed in Figure 4 for both models.

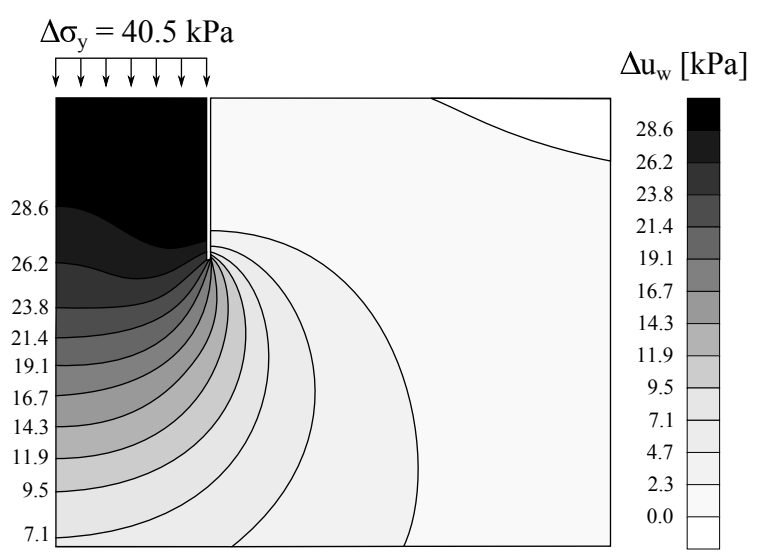

Figure 5: Zoom of distribution of PWP variation $\Delta u_{w}$ around the caisson at the end of the push test. $k=$ $10^{-12} \mathrm{~m}^{2}$. Constitutive law : Prevost model.

Firstly a phase of decreasing ratio holds in Figure 4 because PWP is progressively dissipated in the whole domain (see in Figure 5). As a matter of fact, the PWP increases but slower than the total load. It can be observed that the slope of the curve changes brutally for each simulation. This point corresponds to the maximum mobilised friction along the skirts. Therefore, each increment of total load is fully borne by the soil inside and greater PWP are generated. Afterwards, depending on the permeability, PWP continues increasing or reaches a local maximum than decreases again. The exact evolution depends on a complex alchemy of friction along the skirts, drainage path and rate of loading.

At the beginning, the Prevost model seems to generate lower PWP. This might be explained by another initial stress repartition. However during the loading and especially the first decreasing phase, the PWP generation is higher and as a consequence, the ratio $\Delta u_{w} / \Delta \sigma_{y}$ decreases less rapidly or increases faster afterwards. The reason lies within the contractive behaviour for low $\eta=q / p$ ratios. This can be observed in Figure 6, which depicts $\eta$ repartition around the caisson at the end of the push test for the Prevost model and a permeability of $k=10 \cdot 10^{-12} \mathrm{~m}^{2}$. Every $\eta<\bar{\eta}=0.3$ indicates a contractive zone and therefore PWP generation (if plasticity occurs). It must be pointed out that the soil just under the top of the caisson in Figure 6 has just past the phase transformation line and becomes dilative, which might partially explain the change in tendency of the curve $\Delta u_{w} / \Delta \sigma_{y}$ in Figure 4. 


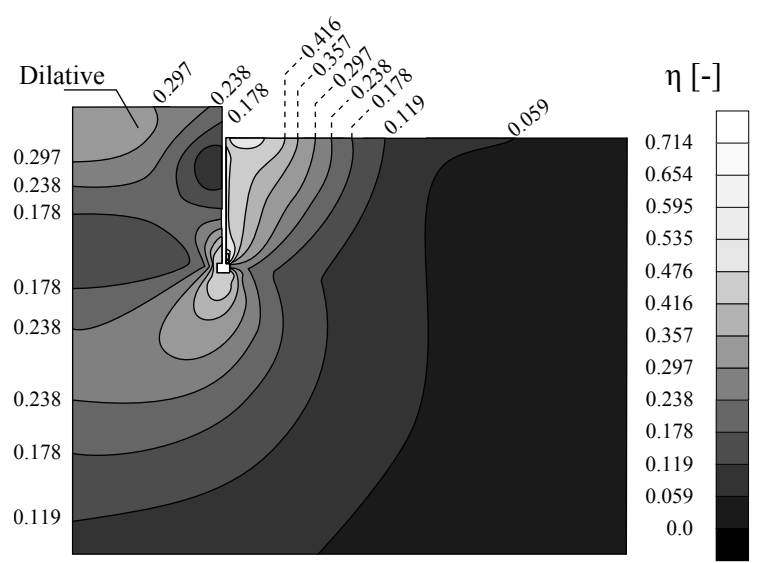

Figure 6: Zoom of distribution of $\eta=q / p$ around the caisson at the end of the push test. $k=10 \cdot 10^{-12} \mathrm{~m}^{2}$. Constitutive law : Prevost model.

Consequences of that behaviour are of uttermost importance. Firstly, the bearing capacity lies within undrained and drained capacity. Depending on the permeability of the soil, a drained design of the caisson should be very conservative. Secondly, the effective stress does not vary a lot, since a significant part of the loading is transferred to the interstitial fluid. Therefore, the settlement is weaker since it depends on the variation of the vertical effective stress.

\subsection{Comparison of signal types}

The time evolution of PWP measured at the top of the caisson is provided in Figure 7 for the first equivalent and the random signals (described in Figure 2). Only the tendency and envelop curves are depicted in order to simplify the reading. It clearly appears that the major part of the loading is sustained by the PWP.

PWP generated during a cycle is most of the time dissipated during the same cycle. However, during higher amplitude cycles, a PWP accumulation occurs during the storm (see in Figure 7), due to the contractive behaviour of the soil around. These overpressures are finally dissipated during low amplitude cycles or consolidation phase.

It must be pointed out that this accumulation reduces the minimum negative PWP that holds during the peak traction event (the difference between $\Delta u_{w}$ and $\Delta \sigma_{y}$ curves is higher in extension than in compression). Therefore, the friction resistance is much more sollicitated.

Tendencies of PWP accumulation are similar for all signals (see in Figure 8). However, the third equivalent signal (maximum amplitude cycles at the end) seems to encounter lower maximum and accumulated PWP. This might be due to the "preparation" of the soil by the small cycles. Indeed the soil has already accumulated plasticity effects and contraction before the high cycles appear and their effect is mitigated. However the difference is too tiny to be peremptorily established.
The peak of PWP accumulation of the pseudo random signal in Figure 7 is narrower than the others. As a matter of fact, the pseudo-random distribution of cycle are favourable to pore pressure dissipation. Indeed high amplitude cycles (favourable to PWP accumulation) and low amplitude cycles (favourable to dissipation) are distributed alternatively whilst they are condensed in equivalent signals.
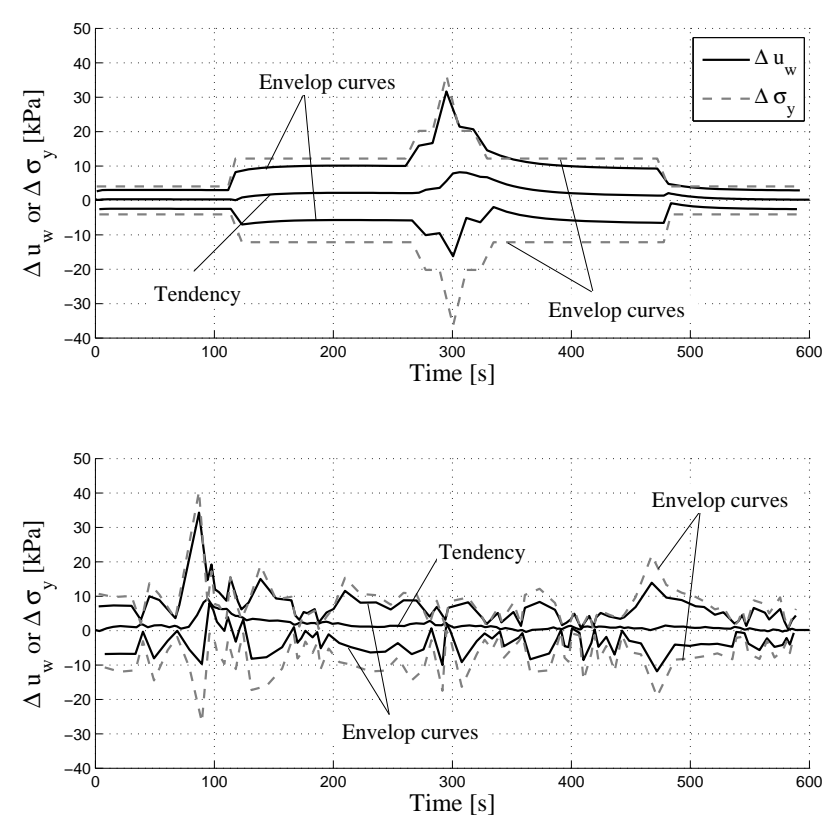

Figure 7: Comparison of envelop curves for PWP and cyclic loading applied to the caisson. The PWP is measured at the top of the caisson for equivalent or pseudo-random loading $\left(\Delta \sigma_{y, \max }=40.5 \mathrm{kPa}\right)$.

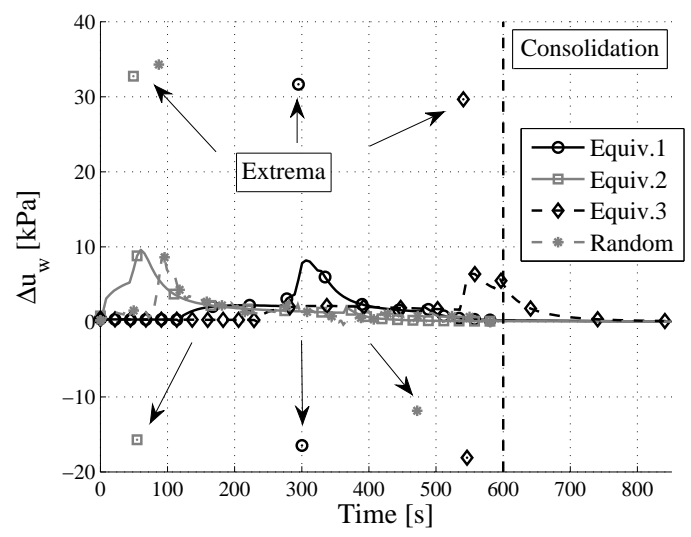

Figure 8: PWP at the top of the caisson for pseudorandom or equivalent loading $\left(\Delta \sigma_{y, \max }=40.5 \mathrm{kPa}\right)$

For the global design, displacement of the rotor of the wind turbine has to be limited, (Senders 2009). Hence displacement of the whole foundation and then of each suction caisson is of great importance. Results for the four loading signals are given in Figure 9 , a positive displacement indicates a settlement. The four signals converge to a quite similar final permanent displacement after dissipation of excess PWP. This outcome involves that the same storm might be simulated by any of the equivalent signal, even if a 


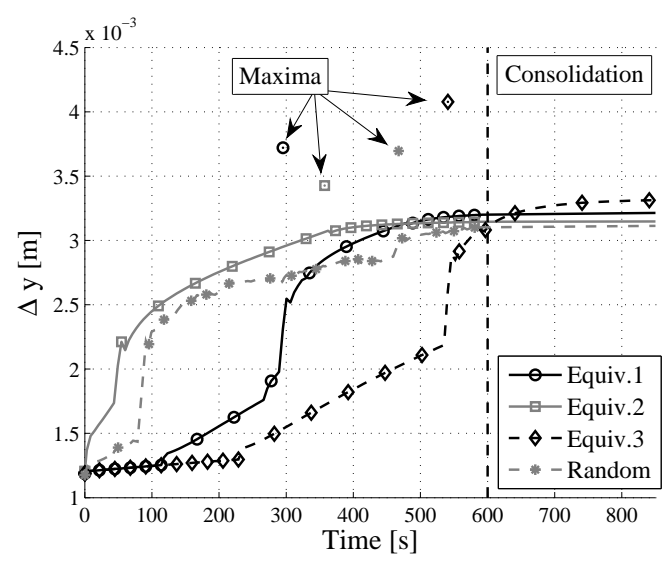

Figure 9: Total vertical displacement of the caisson for pseudo-random or equivalent loading $\left(\Delta \sigma_{y, \max }=\right.$ $40.5 \mathrm{kPa})$

greater number of cycles might affect this conclusion. However, it must be pointed out that the best fit with pseudo-random loading holds for the second equivalent signal, i.e. a signal whose the extreme event is located at the beginning, which is also the case for the pseudo-random loading.

Finally, displacement before and after the storm are provided in Figures 9 and 10 respectively. These figures highlight the deformation of the soil inside the caisson, mainly due to its contractive behaviour. It must be pointed out that this deformation is also strongly coupled to the permeability. Indeed, a lower permeability involves weaker effective stress and therefore weaker solid skeleton deformations.
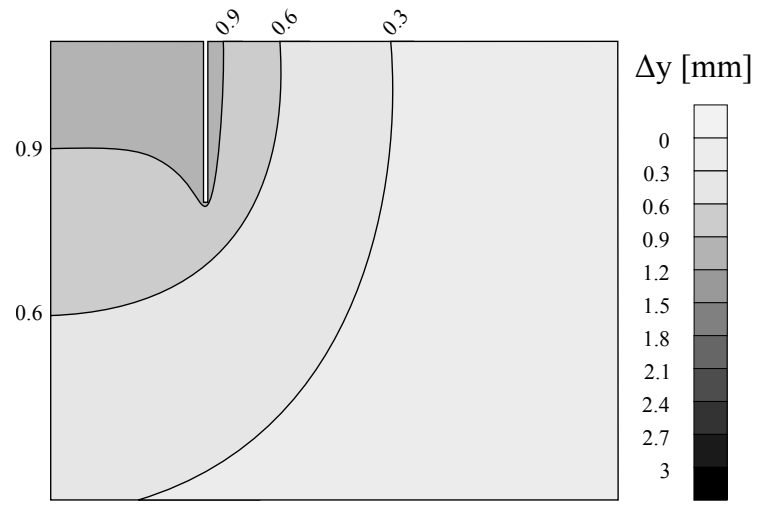

Figure 10: Vertical displacement around the caisson after drained pre-loading. Constitutive law : Prevost model.

\section{CONCLUSIONS}

A fully-coupled transient axisymmetric analysis of a suction caisson in the finite element code LAGAMINE was presented. The partially drained behaviour of such a foundation is highlighted by a monotonic push test. The total load applied is distributed between the soil inside the caisson and a friction resistance along the skirts. The former contribution is mainly equilibrated by the PWP inside the caisson. This overpressure is progressively dissipated in the soil around. During this loading, the soil has mainly a plastic contractive behaviour, which involves more PWP generation and settlement. Therefore this nearly undrained

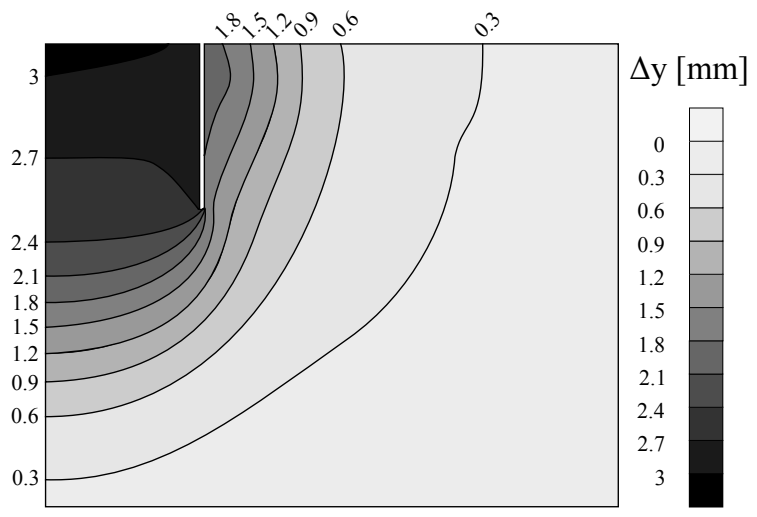

Figure 11: Vertical displacement around the caisson after transient undrained loading . Constitutive law : Prevost model.

behaviour is of uttermost importance since the solid skeleton is less loaded, then settlement is weaker.

Storm can be modelled either by pseudo-random or sinusoidal-equivalent vertical load signals. All the results show a PWP accumulation inside the caisson, due to the contractive behaviour of the soil. However, the pseudo-random load is more prone to dissipate them, due to the alternating series of high and low amplitude cycles, which respectively accumulates and does not accumulate PWP. After consolidation, all the results seem to converge to a very close range of permanent displacement. However the duration of the storm is too short to peremptorily conclude that all signals lead to similar results. The closest results is the signal whose the extreme event is located at the beginning, such as in the pseudo random loading.

\section{REFERENCES}

Byrne, B. W. \& G. T. Houlsby (2002). Experimental investigations of response of suction caissons to transient vertical loading. Journal of Geotechnical and Geoenvironmental Engineering 128(11), 926-939.

Habraken, M., S. Cescotto, \& Q. Banning (1998). Contact Between Deformable Solids: The Fully Coupled Approach. Mathematical and Computer modelling 28(4), 153-169.

Houlsby, G. T., L. B. Ibsen, \& B. W. Byrne (2005). Suction caissons for wind turbines. In Proceedings of the international symposium on frontiers in offshore geotechnics, pp. 75-94.

Ishihara, K., F. Tatsuoka, \& S. Yasuda (1975). Undrained deformation and liquefaction of sand under cyclic stress. Soils and Foundations 15, 29-44.

Kelly, R., G. Houlsby, \& B. Byrne (2006). A comparison of field and laboratory tests of caisson foundations in sand and clay. Géotechnique 56(9), 617-626.

Prevost, J. (1985). A simple plasticity theory for frictional cohesionless soils. Soil Dynamics and Earthquake Engineering 4, 9-17.

Rahman, M., J. R. Booker, \& H. B. Seed (1977). Pore pressure development under offshore gravity structures. Journal of the Geotechnical Engineering Division 103(12), 1419-1436.

Senders, M. (2009). Suction caissons in sand as tripod foundations for offshore wind turbines. Univ. of Western Australia.

Stuyts, B., J. Irvine, \& D. Cathie (2011). Assessing the stability of tripod foundations for offshore wind turbines under cyclic loading. In EURODYN 2011, pp. 3482-3489.

Yang, Z. \& A. Elgamal (2008). Multi-surface cyclic plasticity sand model with lode angle effect. Geotechnical and Geological Engineering 26, 335-348. 